\title{
Racial/Ethnic differences in Metabolic Syndrome in high-income countries: a systematic review protocol
}

Nicholas Kofi Adjei ( $\square$ adjei@uni-bremen.de)

Universitat Bremen

Florence Samkange-Zeeb

Leibniz Institute for Prevention Research and Epidemiology - BIPS, Germany

Mihiretu Kebede

Leibniz Institute for Prevention Research and Epidemiology - BIPS, Germany

Maham Saleem

Leibniz Institute for Prevention Research and Epidemiology - BIPS, Germany

Thomas L Heise

Leibniz Institute for Prevention Research and Epidemiology - BIPS, Germany

Hajo Zeeb

Leibniz Institute for Prevention Research and Epidemiology - BIPS, Germany

\section{Protocol}

Keywords: Metabolic Syndrome (MetS), race, ethnicity, migrants, high-income countries

Posted Date: January 7th, 2020

DOI: https://doi.org/10.21203/rs.2.20156/v1

License: (9) (i) This work is licensed under a Creative Commons Attribution 4.0 International License.

Read Full License

Version of Record: A version of this preprint was published at Systematic Reviews on June 8th, 2020. See the published version at https://doi.org/10.1186/s13643-020-01400-y. 


\section{Abstract}

\section{Background}

Metabolic Syndrome (MetS) is a constellation of several known cardiovascular and type 2 diabetes risk factors, including hyperglycemia, obesity, dyslipidemia and hypertension. While there is some evidence that MetS differ between some population subgroups (i.e., sex, race and ethnicity), not much is known about patterns of MetS among different racial/ethnic groups. The aim of this review is to systematically assess the literature on the prevalence of MetS between racial/ethnic groups, including migrant populations in high-income countries.

\section{Methods}

The preferred reporting items for Systematic Reviews and Meta-Analyses (PRISMA) guidelines will be followed and the following databases searched for relevant studies: MEDLINE, the Social Science Citation Index (SSCl), the Science Citation Index (SCI), the Cumulative Index to Nursing \& Allied Health Literature (CINAHL), and the Cochrane Library databases. A combination of keywords and subject headings will be used in each database to cover all aspects of racial and ethnic differences (i.e. race, ethnicity, minority populations) as well as metabolic syndrome. Cross-sectional, case-control, cohort and ecological studies on MetS that meet the inclusion criteria will be synthesised using narrative synthesis, and the results will be presented using reported outcome statistics (i.e., incidence and prevalence).

\section{Conclusion}

This is the first review that we are aware of to systematically synthesize and collate the available evidence on the frequency, incidence or prevalence of MetS between racial/ethnic groups, including migrant populations. The findings may guide policy formulation and the same time highlight gaps in the literature that future research should address.

\section{Background}

Metabolic Syndrome (MetS) is a clustering of metabolic and physiological abnormalities [1], including hyperglycaemia, obesity, atherogenic dyslipidemia and hypertension [1, 2]. As MetS is not a single pathogenic entity, but rather an array of risk factors for cardiovascular and metabolic diseases [3, 4], various institutions have defined it differently [5-7]. Among these institutions are the World Health Organisation (WHO) [8], International Diabetes Federation (IDF) [6], National Cholesterol Education Program Adult Treatment Panel III (NCEP: ATPIII) [9] and the American Heart Association/National Heart, Lung and Blood Institute (AHA/NLHBI) [5]. Although there are some variations in the definitions of MetS proposed by the named institutions [6], these are minor. At present, the two most widely used definitions are those put forward by the NCEP: ATPIII and IDF [10]. The NCEP: ATPIII definition requires any three of the following five components: 
1. Waist circumference: $\geq 102 \mathrm{~cm}$ for males and $\geq 88 \mathrm{~cm}$ for females

2. Triglycerides: $150 \mathrm{mg} / \mathrm{dl}$ or greater

3. HDL-cholesterol: $<40 \mathrm{mg} / \mathrm{dl}$ in men and $<50 \mathrm{mg} / \mathrm{dl}$ in women

4. Blood pressure: diastolic $\geq 130$ or systolic $\geq 85 \mathrm{mmHg}$

5. Fasting plasma glucose: $110 \mathrm{mg} / \mathrm{dl}$ or greater

The International Diabetes Federation (IDF) on the other hand defined MetS to include central obesity (Waist circumference: $\geq 94 \mathrm{~cm}$ for males and $\geq 80$ for females) as a predominant risk factor along with any two or more of other metabolic risk factors such as raised triglycerides $(\geq 1.7 \mathrm{mmol} / \mathrm{l}(150 \mathrm{mg} / \mathrm{dl}))$ or specific treatment for this lipid abnormality, reduced HDL-cholesterol $(<1.03 \mathrm{mmol} / \mathrm{l}(40 \mathrm{mg} / \mathrm{dl})$ in males and $<1.29 \mathrm{mmol} / \mathrm{l}(50 \mathrm{mg} / \mathrm{dl})$ in females), raised blood pressure (Systolic: $\geq 130 \mathrm{mmHg}$ or Diastolic: $\geq 85 \mathrm{mmHg}$ ) or treatment of previously diagnosed hypertension, and raised fasting plasma glucose $(\geq 5.6 \mathrm{mmol} / \mathrm{l}(100 \mathrm{mg} / \mathrm{dl}))$ or previously diagnosed type 2 diabetes [6].

The worldwide prevalence of MetS among adults is estimated to be $20 \%-25 \%$ [11], but there are country and regional variations [12] depending on the definitions used [10]. The National Health and Nutrition Examination Survey (NHANES) in the US estimated the prevalence of MetS to be $34.5 \%$, based on the NCEP: ATPIII criteria [13]. Australia has a prevalence of 22.1\% using the NCEP:ATPIII definition and $30.7 \%$ using the IDF definition [14]. In Europe, the overall prevalence has been reported to be $24.3 \%$ according to the NCEP:ATPIII definition [15].

MetS and its components have become a public health challenge in high-income countries [16, 17], with their cost to the EU economy, including productivity loss and informal care, being estimated to be about $€ 210$ billion a year [16]. Moreover, there is some evidence that MetS differ between specific populations (i.e., sex, race and ethnicity) within a country $[13,18]$. Prior studies in Europe suggest a higher prevalence of MetS among migrants/ethnic minorities than host populations [19]. In the US, some racial/ethnic differences have also been noted $[20,21]$. Although previous studies suggest that MetS prevalences differ among racial and ethnic groups $[13,18,20,21]$, there has not been any systematic review synthesizing the evidence [4]. The aim of this review is to systematically synthesize the literature on the prevalence of MetS between racial/ethnic groups. The research question that will be addressed is:

To what extent do incidence and prevalence of MetS differ among racial / ethnic groups in high-income countries?

\section{Method}

This systematic review will follow the preferred reporting items for Systematic Reviews and MetaAnalyses (PRISMA) guidelines [22] (see Additional file 1). The review has been registered with PROSPERO (registration number: XXXXXXX).

\section{Inclusion and exclusion criteria}




\section{Study design}

Studies containing quantitative data will be eligible for inclusion. This includes cross-sectional, casecontrol, cohort and ecological study designs. Qualitative studies, opinion studies, case reports and conference presentations will be excluded. All relevant studies will be obtained in order to gain an overview on the incidence and prevalence of MetS between racial/ethnic groups.

\section{Population}

Eligible populations are healthy non-institutionalized adults ( $\geq 18$ years) with self-reported ethnicity/race (e.g., Black, White, Hispanic/Latina and Asian) in high-income countries, as classified by the Organisation for Economic Co-operation and Development (OECD) [23]. Studies confined to clinically selected patients will be excluded. All accepted definitions of MetS will be eligible for inclusion.

\section{Exposure}

The exposure of interest will be race /ethnicity. Studies to be included must compare two or more racial/ethnic groups. However, a homogeneous population with diverse tribal groups will be excluded. Those comparing migrants and host populations will be included.

\section{Comparator/control}

The comparator will be ethnic "majority" groups. For example, in the US, the majority ethnic group would be "White" while the minority ethnic group would be "Black", "Hispanic", "Asian" and "other" ethnic backgrounds [24]. In Europe, the minority ethnic group will generally also include people with a migration background [25].

\section{Outcomes}

Studies will be included if they contain any measure of incidence and prevalence of MetS among different racial/ ethnic groups (primary outcomes). The specific components of MetS (i.e., central obesity, raised blood pressure, hyperglycaemia and dyslipidaemia) will be additional outcomes of this study (secondary outcomes). Studies not reporting on the primary outcomes as defined here will be excluded.

\section{Search strategy and study selection}

All articles will be selected based on the research objective and the predefined inclusion criteria. The search will include peer reviewed literature that is published in electronic databases. The following databases will be searched: MEDLINE, the Social Science Citation Index (SSCI), the Science Citation Index (SCI), the Cumulative Index to Nursing \& Allied Health Literature (CINAHL), and the Cochrane Library databases will be searched to identify articles published up to November 2019. A combination of keywords and subject headings will be used in each database to cover all aspects of racial and ethnic differences (i.e. race, ethnicity, minority populations) as well as metabolic syndrome. We will also search the reference lists of included articles to identify and include additional citations not found in the initial 
search. Books or grey literature will be excluded. The search will not be restricted by language. The search strategy has been piloted by the author team and successfully retrieved pre-selected records which are relevant to the review scope. The search will be modified according to the requirements of the other bibliographic databases (Table 1).

Table 1. Medline Search Strategy

\begin{tabular}{|c|c|}
\hline \multicolumn{2}{|l|}{ Line } \\
\hline 1 & ((race* or racial or ethnic* or ethnicit*) adj5 minorit*).ti,ab. \\
\hline 2 & $\begin{array}{l}\text { ((raci* or race or ethnic* or minorit* or immigra* or emigra* or migrant* or } \\
\text { migration) adj5 (group* or communit* or population*)).ti,ab. }\end{array}$ \\
\hline 3 & exp minority groups/ \\
\hline 4 & exp minority health/ \\
\hline 5 & exp ethnic groups/ \\
\hline 6 & exp "emigrants and immigrants"/ \\
\hline 7 & or $/ 1-6$ \\
\hline 8 & METS.ti,ab. \\
\hline 9 & metabolic syndrome.ti,ab. \\
\hline 10 & exp metabolic syndrome/ \\
\hline 11 & or $/ 8-10$ \\
\hline 12 & 7 and 11 \\
\hline 13 & $\begin{array}{l}\text { ((race* or racial or ethnic* or raci* or immigra* or emigra* or migrant* or } \\
\text { migration or minorit*) adj15 (METS or metabolic syndrome)).ti,ab. }\end{array}$ \\
\hline 14 & 12 or 13 \\
\hline
\end{tabular}

Search results will be uploaded into Endnote and duplicates will be removed. The citations will then be imported into Covidence (www.covidence.org), a web-based software that aids the management of systematic reviews. This software will further be used for abstract and title screening. Two reviewers will screen potential articles by titles and /or abstracts to identify studies that meet the inclusion criteria. The same reviewers will independently screen the full texts of all potentially eligible studies and compare their findings. Any disagreements between the two review authors will be resolved through consensus and where necessary, a third reviewer will be involved to resolve contradictory judgements.

\section{Data extraction}

Data extraction will be done using MS Excel and the following details will be extracted from each study: i) details of the study (first author's last name, year of publication, country), ii) study design (study design, sample size, sampling method, ethnic group, age and gender of participants), iii) metabolic syndrome definition criteria, iv) metabolic syndrome frequency, incidence and prevalence data for all adults. A second reviewer will check the extracted data against the original data. In case of missing or incomplete data, authors will be contacted to assist in the process.

\section{Quality assessment and risk of bias}


The risk of bias in the included studies will assessed by two review authors using the Effective Public Health Practice Project (EPHPP) assessment tool for quantitative studies [26]. The EPHPP assess different components of study validity: study design, confounders, selection bias, binding, data collection method and dropouts. The overall methodological quality will be rated as strong, moderate or weak, and where there are differences in opinion, a third reviewer will be consulted.

\section{Data synthesis and analysis}

We anticipate a sizable statistical heterogeneity across studies [27], hence it may be difficult to conduct a meta-analysis. Should this be the case, we will describe and synthesize the data using narrative synthesis [28]. This approach will synthesize the evidence for the included studies in accordance with the objectives of the study. The narrative synthesis will highlight key findings of the included studies [28] and the results will be presented using outcome statistics (i.e., prevalence and incidence). In addition, definitions of MetS, distribution of studies across geographical locations and publication years will be presented in the summary of findings table.

\section{Discussion}

MetS and its components, namely, central obesity, raised blood pressure, hyperglycaemia and dyslipidaemia [1, 2, 29] have been identified as risk factors for type 2 diabetes [30, 31], cardiovascular diseases [3] and coronary heart disease related mortality [32]. To our knowledge, this will be the first review to systematically synthesize and collate the available evidence on the incidence of prevalence of MetS in different racial/ethnic groups, including migrant populations. The changing population structure in high-income countries [33], which may be due to high influx of international migrants [34] allows for a sound assessment of MetS. The findings will provide an opportunity for interventions to avert the development of cardiovascular diseases among certain racial/ethnic groups [35], especially ethnic minorities [25]. It may further guide policy formulation and also highlight gaps in the literature that need to be considered in future research.

\section{Abbreviations}

CINAHL - Cumulative Index to Nursing \& Allied Health Literature

EPHPP - Effective Public Health Practice Project

IDF - International Diabetes Federation

MetS - Metabolic Syndrome

NCEP: ATPIII - National Cholesterol Education Program Adult Treatment Panel III

NHANES - National Health and Nutrition Examination Survey 
OECD - Organisation for Economic Co-operation and Development

PRISMA - Preferred Reporting Items for Systematic Reviews and Meta-Analyses

SCl - Science Citation Index

SSCI - Social Science Citation Index

WHO - World Health Organization

\section{Declarations}

\section{Acknowledgments}

The authors would like to acknowledge the help of Ms. Lara Christianson for selecting appropriate databases and compiling search items for this study.

\section{Funding}

This work was supported by the Leibniz Institute for Prevention Research and Epidemiology - BIPS, Germany Intramural Research Fund.

\section{Availability of data and material}

Not applicable

\section{Author Contribution}

NKA and $\mathrm{HZ}$ conceived the initial idea for the study. NKA wrote the protocol with input from MS. NKA, HZ, MS, FSZ, MK and TH critically appraised the protocol manuscript. NKA will act a first reviewer, and MS, FZ-K, MK and TH will act as second reviewers in the review. All authors approved the final version of the manuscript.

\section{Competing interests}

The authors declare that they have no competing interests.

\section{Consent for publication}

Not applicable

\section{Ethics approval and consent to participate}

Not applicable

\section{References}


1. Alberti, K.G.M., P. Zimmet, and J. Shaw, The metabolic syndrome-a new worldwide definition. The Lancet, 2005. 366(9491): p. 1059-1062.

2. Grundy, S.M., et al., Diagnosis and management of the metabolic syndrome: an American Heart Association/National Heart, Lung, and Blood Institute scientific statement. Circulation, 2005. 112(17): p. 2735-2752.

3. Galassi, A., K. Reynolds, and J. He, Metabolic syndrome and risk of cardiovascular disease: a metaanalysis. The American journal of medicine, 2006. 119(10): p. 812-819.

4. Mottillo, S., et al., The metabolic syndrome and cardiovascular risk: a systematic review and metaanalysis. Journal of the American College of Cardiology, 2010. 56(14): p. 1113-1132.

5. Grundy, S., American heart association; national heart, lung, and blood institute. Diagnosis and management of the metabolic syndrome: an American heart association/national heart, lung, and blood institute scientific statement. Circulation, 2005. 112: p. 2735-2752.

6. Alberti, K.G.M.M., P. Zimmet, and J. Shaw, Metabolic syndrome-a new world-wide definition. A consensus statement from the international diabetes federation. Diabetic medicine, 2006. 23(5): p. 469-480.

7. Balkau, B., Comment on the provisional report from the WHO consultation. European Group for the Study of Insulin Resistance (EGIR). Diabet med, 1999. 16: p. 442-443.

8. Alberti, K.G.M.M. and P.Z. Zimmet, Definition, diagnosis and classification of diabetes mellitus and its complications. Part 1: diagnosis and classification of diabetes mellitus. Provisional report of a WHO consultation. Diabetic medicine, 1998. 15(7): p. 539-553.

9. National Institutes of Health. Third report of the National Cholesterol Education Program (NCEP) Expert Panel on detection, evaluation, and treatment of high blood cholesterol in adults (Adult Treatment Panel III). 2002: International Medical Pub.

10. Kassi, E., et al., Metabolic syndrome: definitions and controversies. BMC medicine, 2011. 9(1): p. 48.

11. Mulder, R.L., et al., Hepatic late adverse effects after antineoplastic treatment for childhood cancer. Cochrane Database of Systematic Reviews, 2019. 4: p. CD008205.

12. Cameron, A.J., J.E. Shaw, and P.Z. Zimmet, The metabolic syndrome: prevalence in worldwide populations. Endocrinology and Metabolism Clinics, 2004. 33(2): p. 351-375.

13. Ford, E.S., Prevalence of the metabolic syndrome defined by the International Diabetes Federation among adults in the US. Diabetes care, 2005. 28(11): p. 2745-2749.

14. Cameron, A.J., et al., The metabolic syndrome in Australia: prevalence using four definitions. Diabetes research and clinical practice, 2007. 77(3): p. 471-478.

15. Scuteri, A., et al., Metabolic syndrome across Europe: different clusters of risk factors. European journal of preventive cardiology, 2015. 22(4): p. 486-491.

16. Wilkins, E., et al., European cardiovascular disease statistics 2017. European Heart Network, Brussels.

17. Murray, C.J., et al., Disability-adjusted life years (DALYs) for 291 diseases and injuries in 21 regions, 1990-2010: a systematic analysis for the Global Burden of Disease Study 2010. The lancet, 2012. 
380(9859): p. 2197-2223.

18. Rampal, S., et al., Ethnic differences in the prevalence of metabolic syndrome: results from a multiethnic population-based survey in Malaysia. PloS one, 2012. 7(9): p. e46365.

19. van der Linden, E.L., et al., The prevalence of metabolic syndrome among Ghanaian migrants and their homeland counterparts: the Research on Obesity and type 2 Diabetes among African Migrants (RODAM) study. European Journal of Public Health, 2019.

20. Beltrán-Sánchez, H., et al., Prevalence and trends of metabolic syndrome in the adult US population, 1999-2010. Journal of the American College of Cardiology, 2013. 62(8): p. 697-703.

21. Ford, E.S., W.H. Giles, and W.H. Dietz, Prevalence of the metabolic syndrome among US adults: findings from the third National Health and Nutrition Examination Survey. Jama, 2002. 287(3): p. 356-359.

22. Moher, D., et al., Preferred reporting items for systematic reviews and meta-analyses: the PRISMA statement. Annals of internal medicine, 2009. 151(4): p. 264-269.

23. Woodward, R., The organisation for economic co-operation and development (OECD). 2009: Routledge.

24. Yanow, D., Constructing race and ethnicity in America: Category-making in public policy and administration. 2015: Routledge.

25. van der Linden, E.L., et al., The prevalence of metabolic syndrome among Ghanaian migrants and their homeland counterparts: the Research on Obesity and type 2 Diabetes among African Migrants (RODAM) study. European Journal of Public Health, 2019. 29(5): p. 906-913.

26. Armijo-Olivo, S., et al., Assessment of study quality for systematic reviews: a comparison of the Cochrane Collaboration Risk of Bias Tool and the Effective Public Health Practice Project Quality Assessment Tool: methodological research. Journal of evaluation in clinical practice, 2012. 18(1): p. 12-18.

27. Hoffmann, F., et al., An observational study found large methodological heterogeneity in systematic reviews addressing prevalence and cumulative incidence. Journal of Clinical Epidemiology, 2019.

28. Popay, J., et al., Guidance on the conduct of narrative synthesis in systematic reviews. A product from the ESRC methods programme Version, 2006. 1: p. b92.

29. Ahrens, W., et al., Metabolic syndrome in young children: definitions and results of the IDEFICS study. International journal of obesity, 2014. 38(S2): p. S4.

30. Haffner, S.M., et al., Prospective analysis of the insulin-resistance syndrome (syndrome X). Diabetes, 1992. 41(6): p. 715-722.

31. Isomaa, B., et al., Cardiovascular morbidity and mortality associated with the metabolic syndrome. Diabetes care, 2001. 24(4): p. 683-689.

32. Malik, S., et al., Impact of the metabolic syndrome on mortality from coronary heart disease, cardiovascular disease, and all causes in United States adults. Circulation, 2004. 110(10): p. 12451250. 
33. Rechel, B., et al., Migration and health in an increasingly diverse Europe. The Lancet, 2013. 381(9873): p. 1235-1245.

34. Hugo, G. and R. Morén-Alegret, International migration to non-metropolitan areas of high income countries: editorial introduction. Population, Space and Place, 2008. 14(6): p. 473-477.

35. Lip, G., et al., Ethnicity and cardiovascular disease prevention in the United Kingdom: a practical approach to management. Journal of human hypertension, 2007. 21(3): p. 183. 